\section{Omics analyses of tumour immunity}

the underlying
genomic
alterations
might provide
valuable
biomarkers
or targets
for immuno-
therapy

Tumour tissue samples typically comprise a mix of tumour cells and non-tumour cells, including stromal cells and lymphocytes. All of these cell types will be represented in bulk molecular analyses of these samples, which complicates inferences about the tumour cells themselves but provides a valuable window into the state of the tumour microenvironment. A new omics analysis of heterogeneous tumour samples identifies various putative molecular determinants of the anti-tumour immune response.

Anti-tumour activity of the immune system is an important intrinsic tumour suppressor mechanism that various tumour immunotherapy approaches aim to boost. To gain mechanistic insights into the determinants of anti-tumour responses, Rooney et al. analysed DNA sequence, copy number and transcriptomic data sets from thousands of solid tissue biopsy samples for 18 tumour types in The Cancer Genome Atlas (TCGA). They measured the RNA expression levels

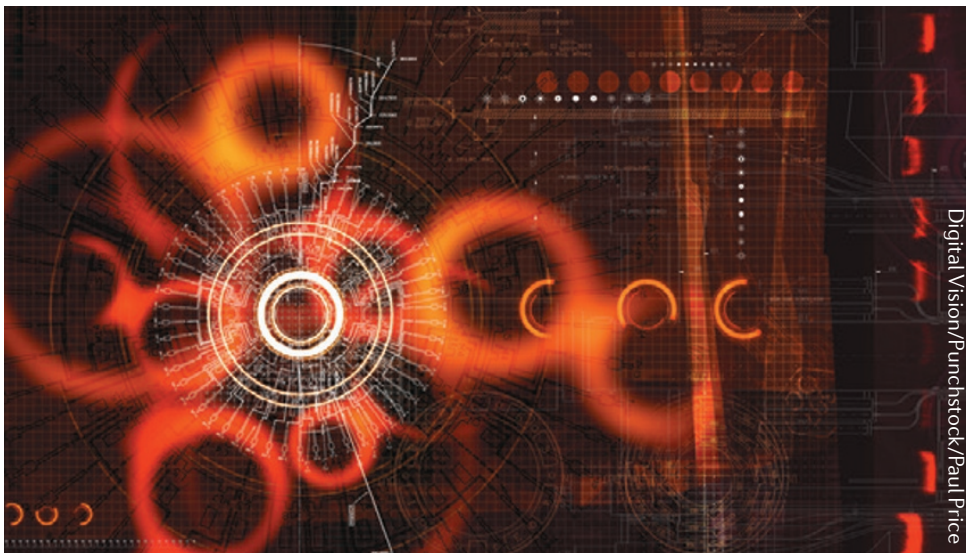

of key immune effectors, granzyme A (GZMA) and perforin 1 (PRF1), as markers of the cytolytic activity of cytotoxic T lymphocytes and natural killer cells towards the tumours they were infiltrating.

Although expression of these markers varied widely across different tumour types, the researchers found correlations between immune cytolytic activity and numerous molecular features of tumours. Cytolytic activity correlated with somatic mutations: both the overall tumour mutation load and the number of mutations that were probable neo-epitopes (that is, protein-coding mutations that are predicted to be presented to the immune system as peptides on the patient's particular major histocompatibility complexes). Additionally, cytolytic activity correlated with infection by different tumour-associated viruses and with the reactivation of endogenous retroviruses in tumours. Hence, tumour-expressed antigens (from somatic mutations or viruses) seem to be important for enabling the immune system to detect and target tumour cells.

The investigators further identified mutations of various cancer driver genes and mutations causing defective antigen presentation or apoptosis that were associated with increased cytolytic activity. They propose that these mutations in tumour cell clones facilitate tumour cell survival and persistence despite sustained cytolytic activity in the microenvironment. In addition, various copy number alterations in immune regulatory genes (such as PDL1, PDL2 and the ALOX gene family) also correlated with cytolytic activity.

Finally, the authors demonstrated that the cytolytic activity was associated with a modest survival benefit to patients; hence, some of the underlying genomic alterations might provide valuable biomarkers or targets for immunotherapy.

Darren J. Burgess

ORIGINAL RESEARCH PAPER Rooney, M. S. et al. Molecular and genetic properties of tumors associated with local immune cytolytic activity. Cell 160, 48-61 (2015) 